\title{
Generalities About Caring Mental Illness and Impact on Family Caregivers in Chinese Community: A Ten Years Narrative Review Study
}

\section{Nkundimana Balthazar \\ $\mathrm{Ph}$. D scholar, Department of psychiatry and mental health, The first affiliated hospital of Chongqing Medical University, Chongqing 400016, People's Republic of China, China}

\section{Xu Xiaoming \\ The first affiliated hospital of Chongqing Medical University, Chongqing 400016, People’s Republic of China, China}

\section{Prof. Kuang $\mathbf{L i}^{*}$}

Professor, Department of psychiatry and mental health, The first affiliated hospital of Chongqing Medical University, Chongqing 400016, People's Republic of China, China Email: kuang0308@163.com

\section{Fei Gao}

The first affiliated hospital of Chongqing Medical University, Chongqing 400016, People's Republic of China, China

\section{Jiglan He}

The first affiliated hospital of Chongqing Medical University, Chongqing 400016, People's Republic of China, China

\section{Hui Pang}

The first affiliated hospital of Chongqing Medical University, Chongqing 400016, People's Republic of China

\section{Yuxue Zhang}

The first affiliated hospital of Chongqing Medical University, Chongqing 400016, People's Republic of China, China

\section{Zhou Yi}

The first affiliated hospital of Chongqing Medical University, Chongqing 400016, People’s Republic of China, China

\section{Sanjay Kumar Sah}

$\mathrm{Ph}$. D scholar, Joint International Research Laboratory of Reproduction and Development, Chongqing Medical University, Chongqing 400016, People's Republic of China, China

\begin{abstract}
Background: Worldwide, the burden of mental health is increasing day by day. The WHO alerts countries that diseases caused by mental health will occupy the second rank on the rest of non-communicable diseases. The increase of mental diseases is always associated with the increase of multiple challenges in primary caregivers who are mostly family members of the patient. China is one of nations with high prevalence of mental health disorders. Like many other countries caregiving mental health in china would have challenges and opportunities. This study aimed to narratively combine studies on family caregivers of psychiatric illness in China thereby making an objective conclusion Methods: A matrix method was narratively used in this study. Studies conducted in Chinese community which includes studies in main land China, Hong Kong and Taiwan conducted in the period between 2008-2019 were considered. 47 studies were considered for analysis and five main themes were found for consideration. Results: The findings in this study are summarized in the following themes 1. High burden and burnout of care and influencing factors, 2. The high stigma of caregivers 3. The low quality of life of caregivers 4. the social support. 5. Care and intervention for caregivers of psychiatric disorders. Conclusion: caregivers of psychiatric illness in Chinese community face challenges that are in some cases different to the rest of the world however there are many similarities with the rest of the world that are found. To overcome these challenges, all parties are called to play its assigned role in the eradication of these challenges. There is a need of strengthening the available opportunities and the common understanding of the problem is required in Chinese community.
\end{abstract}

Keywords: Psychiatric patient; Mental illness; Caregivers; China. 


\section{Introduction}

The incidence of mental health and psycho-behavioral problems have increased since last few decades. So far the disease burden caused by mental disorders occupy the average prevalence of approximately $14 \%$ of the rest of non-communicable diseases, making mental disorder the second on the global diseases burden [1]. The disease burden caused by mental health in china counts for $13 \%$ of non-communicable diseases burden [2]. The increase of mental health patients increases the number of caregivers. Caregivers of psychiatric patients are mostly family members of the patient especially in Asian countries like china where at least $80 \%$ of psychiatric patients get support from their families [3]. Family caregivers have started to get involved in caregiving activities since last 50 years when psychiatric care shifted from hospital based to community based in the movement called deinstitutionalization [4]. Different studies reported that both patients and their caregivers are constantly affected by changes resulting from diseases and its treatment. Especially when the disease is running in the family, the family caregiver can face the caregiving challenges such as reduction of the level of daily performance, the ability of family members, destruction of emotional system and communication structure of the family, problems of relationships among members, financial and economic problems, reduced social interactions of the family, changes in roles, reduced life expectancy, and emergence of some psychological symptoms such as anger, feeling of guilty, grief, and even denial $[5,6]$. In many countries, caregivers of psychiatric patients are left without adequate social support and other needed assistance, while some consider them as hidden patients and the lack of these may have a direct impact on patients care and treatment outcome [7]. In the traditional Chinese community including Taiwan, Hong Kong and Mainland China mental illness was considered as a punishment for misdeeds done by individuals or their families. Thus mental illness is generally viewed as individual or family problem later than a problem of general society [8]. Because of this mentality, to avoid stigma and other problems related to the presence of psychiatric patient in family, some family caregivers in Chinese society and some other society choose to keep secret when a family member is mentally ill rather than seek for help [9]. Since last decades Chinese believes and attitudes toward mental health have begun to change. both territories of Chinese community Taiwan ,Hong Kong and Mainland China In 1980s have started to implement the deinstitutionalization policies and other mental health care policies with aim of enabling people with mental health problems to integrate into the society and lead normal life $[10,11]$. Since then many psychiatric patients are following treatment while living mainly with their families and other informal caregivers. Mental healthcare policies have been initiated in china focusing on the patients in general and the sustainability of Chinese mental healthcare in particular. Yet different studies and programs were conducted on caregiving and its related issues in china [12-14].

Numerous studies on caregiving mental illness have been conducted in china. Different aspects of caregiving mental illness have been explored year by year since last decades until today.

A comprehensive overview of studies in this issue is required for at least each ten years to determine challenges and opportunities in the field of caregiving mental illness and mental healthcare in general. Therefore, in this study, scientific evidences and other relevant documents were reviewed to capture the image or an understanding of caregivers of patients with mental health problems in the Chinese community context.

The main objective of this study was to narratively combine studies on caregiving psychiatric illness conducted in Chinese community. The study has then explored studies conducted on caregivers of psychiatric illness in Mainland China, Hong Kong and Taiwan. In the period between 2008-2019.

\section{Materials and Method}

This is a narrative review study. To achieve the study objectives, we used a matrix method. According to Horey and Canty [15]. A review study should contain summaries of previous findings in the literature review of a given topic. In any field the existence of information is both an opportunity and challenge therefore there is a need of a systematic method of review of these information to overcome challenges.

By referring to this point we designed our study as follow; Initially a team composed by mental health workers, $\mathrm{PhD}$ students in psychiatry and mental health and faculty member of psychiatric department of the first affiliated hospital of Chongqing medical university. Literature search carried out by the first author and other authors. The extracted documents were reviewed by each member in the research group independently to include relevancy and appropriate in the study.

The search took place in electronic database NCBI; PUBMED, PUBMED advanced, Google scholar. Web of science and google search. we searched published articles from 2008 to 2019 we used the following key words for search; caregivers and psychiatric patients, families of psychiatric patients, China, Chinese community, Mainland China, Taiwan and Hong Kong Results were combined using the "AND" Boolean operator to ensure inclusion of at least one search terms from each of the four categories. Retrieved articles were screened based on the inclusion and exclusion criteria.

Inclusion criteria in this study included studies focusing on caregivers of psychiatric patients in Chinese society. Participants were informal caregivers including family caregivers of patients with mental disorders of all ages. Both qualitative and quantitative studies were included in this study. Exclusion criteria included book chapters preliminary or pilot studies, studies with no primary focus on the caregivers and focus on patient review article were also excluded. All studies with relevant titles and abstracts were selected for the analysis and were subjected to the critical appraisal which involved assessing their methods and results sections in order to find out their strengths and weakness as well as their merit criteria to be considered to the review question. After applying inclusion and exclusion criteria a total of 47 were found to meet requirements of inclusion for the final review. 


\section{Sumerianz Journal of Medical and Healthcare}

All considered articles were read several times by the first author and reviewed by the other authors in the research team to get a deeper understanding of the studies. Data were extracted based on the year of publication and study settings. Core features of analysis were 1 . Whether study focused specifically on issues regarding caregiving psychiatry patients in Chinese society, whether the study focused primary on caregivers' issues related to the caregiving role such as challenges or problems encountered by family caregivers or other informal caregivers of mental disorders in Chinese society 2. Whether the study focused specifically on the family caregiver or both the caregiver and the patient 3 . The outcome measures, 4 . The used methods, the content and results of the study and year of publication of the study.

Based on the common meanings and central issues all studies that met criteria were organized and integrated as categories and themes.

\section{Results}

After completing the review process, data were extracted as codes of topics relevant to the review question and were qualitatively categorized by research team members to find main themes the generalities about caregiving psychiatric disorders in Chinese community were specified in the emerging following themes: 1. High burden and burnout of care and influencing factors, 2 . The high stigma of caregivers 3 . The low quality of life of caregivers 4 . the social support in caregivers 5. Intervention and care for caregivers of psychiatric disorders.

\subsection{High Burden and Burnout of Care and Influencing Factors}

As reported by many studies, one of the most frequent issue that occupy a big challenge of caregiving mental health illness in Chinese community is the caregivers' burden and the burnout [16]. Explored and compared the burden and its severity in two different groups of schizophrenia and bipolar disorder caregivers. Generally, the high burden was seen in both groups either the schizophrenic caregivers' group as well as the bipodal disorder caregivers' group. As these authors reported each group presented dominating factors that were associated with the burden and burnout, for instance caregivers of perceptions of violent behavior and suicidal risk among caregivers of patients with bipolar disorders group were higher than for caregivers of patients with schizophrenia ,making the he higher burnout within the group of bipolar disorders as compared to the schizophrenia caregivers group [16].

Similar, another study by Zhou, et al. [17]. The burden by comparing two groups of caregivers of schizophrenic patients who are or not living with their families. The study aimed to compare the burnout and burden between the two groups. This study has shown that the burnout and burden was higher in the group that is living with the patient home lather then the group of patients who are living in the intuitions. A multiple regression analysis reported that $6.7 \%, 8.3 \%$ and $6.7 \%$ of the variance in distress, disrupted routines and helpfulness. However, the group of caregivers with patients who are living in institutions also present a kind of burden and burnout in this study. The concluding remarks of requested the general community to give support to this group of caregivers with schizophrenic patients that are living with their community [17].

The burden of caregivers was also identified to be high as compared the psychiatric illness and non-psychiatric illness .In a group of Dementia caregivers and group of Alzhemer caregivers, the burden was found in both groups with higher rate in the groups of Dementia the influencing factors in the group of Dementia caregivers were the severity of neuropsychiatric symptoms and behavioral disturbance of dementia patients [18]. In Beijing the group of caregivers of veteran with dementia has also reported high caregivers burden and burnout the identified influencing factor for the high and burnout burden within these caregivers was mainly was the length daily time spent with the patient [19].

Not only in schizophrenia and or dementia but also caregivers of OCD have reported the burden in all of its form together with the burnout the subjective and objective burden were found in a group of caregivers of OCD on a high rate approximately $99 \%$ of study subject reported the subjective burden and $49.8 \%$ reported subjective burden in the same study the General Assessment function was 41.5\% [20].

The assessment of burden as associated with other factors such as personality the coping style and family functioning was conducted by $\mathrm{Yu}$, et al. [21]. In this this study the high level of burden was directly associated with personality traits especially neuroticism personality traits and the coping style (diathesis), and the family functioning was also found to be influencing factor of high burden and burnout [21].

Another study on schizophrenic patients' caregivers in Guangzhou has found the high burden among caregivers. This study state that the length of staying is directly linked with high burden of caregivers. The authors reported that the long stay of the patient in the hospitals and psychiatric centers plays a role in caregivers' high burden and distress [22].

In rural china, a study which took a sample 327 primary caregivers of schizophrenic patients reported the incidence of $52 \%$ of burden in caregivers the financial burden scores $76 \%$ and was the commonest burden where disruption of family interaction was 37\%. This study reported that the causing factors for high level of burden were gender, where female reported high significant difference of caregiver's burden as compared to male. The other factors were time of hospital admission of the patient where more that 3 admissions were associated with the high caregiver's burden, the level of education, the additional dependents and the higher care network [23].

Similar, to this previous study, again $\mathrm{Yu}$, et al. [24]. Reported the burden and burnout in other study. In their these authors found that $84 \%$ of caregivers. $45.9 \%$ were presenting anxiety while $45,4 \%$ reported depression. And the majority were presenting other physical illness $(67 \%)$ and they have been caregivers for at least 10 years $(74.3 \%)$ [24]. 


\section{Sumerianz Journal of Medical and Healthcare}

Always in rural china, Yu, et al. [21]. Have linked the high burden and distress with gender differences. Caring for male patient was directly linked with high burden and distress. The female caregivers were seen to be significantly vulnerable of presenting higher burden in all of its form as compared to male caregivers. In summary this study found that the anxiety was higher in caregivers of male patients than caregivers of female patients $(52.7 \%$ vs $38.1 \%, \mathrm{P}=0.012)$; female caregivers were more likely to suffer from depression $(51.2 \%$ vs $38.6 \%, \mathrm{P}=0.031)$ and anxiety $(51.6 \%$ vs $38.1 \%, \mathrm{P}=0.020)$ than male caregivers [21].

A qualitative study in Beijing by Chen, et al. [25]. Most Participants caregivers of schizophrenia reported that they were suffering from heavy life burdens. The economic and daily housework burdens, limited social communication and psychological distress were the predominant factors of high burden. In addition, these respondents reported that they were experiencing negative aspects with respect to social support [25].

The combination of these studies shows that the high caregiver's burden and burnout remains a challenge in caregiving psychiatric illness in Chinese community. Factors that are associated with the caregiver's burden were identified in most of studies conducted on the same topic in last ten years. Apparently, the associated factors still vague and ambiguous. However, the concerned people on this field should consider the currently findings and a prevalence study would be advantageous in this domain.

\subsection{The High Stigma of Caregivers}

Another found element in the caregiving psychiatric illness in Chinese community is the stigma. The stigma remains challenge not only in psychiatric patients but also in their caregivers. It has been an issue of concern for many researchers of caring mental health in Chinese society. And it was identified in numerous studies and mostly presented in caregivers at the same time as patients. For instance, Wong, et al. [26]. Identified the stigma within both schizophrenic patients and their caregivers. According to the authors' findings, persons with schizophrenia and their caregivers both presented internalized negative valuation from their social networks and reduced engagement in the community. In this study patients with schizophrenia expressed a sense of shame and inferiority, spoke about being a burden to their family, and expressed self-disappointment as a result of having a psychiatric diagnosis. Family dyads varied in the extent that internalized stigma was experienced patients and caregivers. Apart from stigma caregivers have reported a high level of emotional distress because of mental illness in the family [26].

The characteristic of stigma as the feeling of shame within caregivers was also presented by caregivers in a study of schizophrenia caregivers by Huang, et al. [27]. Respondents in this study reported the stigma that was linked to caregiving a psychiatric patient they also presented that their stigma affects their coping strategies the influencing factors could be seen in their kind behaviors such us traditional help-seeking behaviors feeling of low social status [27].

A study that involved schizophrenic caregivers has also reported the stigma in caregivers. As reported by Hsiao, et al. [28]. The stigma was presented on high rate among caregivers. The same study also has reported the link between caregiving stigma and the low quality of life as well as the household income. The family centered care was also evaluated in this study and was found to have an influence on the caregivers' stigma [28].

Chen, et al. [29]. Have evaluated the stigma in all its form in caregivers as well as patients they have then linked it with other psychological problems within caregivers. Caregivers' affiliate stigma was significantly associated with higher levels of stress, and symptoms of depression and anxiety. And Affiliate stigma did not correlate with perceived public stigma and "face concern" in caregivers. Additionally, Caregivers with greater degree of affiliate stigma experience increased stress and emotional distress [29].

\subsection{The Low Quality of Life Associated to Caregiving}

One of main focus on studies of caregivers of psychiatric patients in Chinese society in the last ten years was the quality of life among caregivers. The quality of live was found to be a challenging issue in the group of psychiatric patients and their caregivers as well Guan, et al. [30]. Studied the quality of life (QoL) within dived groups of patients and their caregivers. The hospitalized group and its caregivers and the community living group with its caregivers. The results reported that community samples had a significantly lower QOL score. The QOL score for the social relationship's domain of the hospitalized patients' caregivers was significantly higher than that of the caregivers of community patients $(\mathrm{P}=0.019)$ [30].

In Hong Kong the quality of life of schizophrenia caregivers was found in a large number of caregivers. The findings indicate that caregivers have significant poor quality of live as compared to the general population. The results in this study indicated that the main influencing factor in low quality of life was the age where younger caregivers presented a significant difference in QOL as compared to adults caregivers, the length of chronicity of the illness, the difficult in handling negative symptoms, the perception of insufficient mental health services and the lower educational level of caregivers [31].

\subsection{The Social Support}

The issue of social support poses a problem in caring mental health illness in Chinese community. Patients and caregivers of mental illness with social support could be associated with the quality of life the burden the stigma and other related problems that occurs when caring psychiatric illness. The literature show that authors interested in caregiving mental illness in China tried to focus in this matter. Studies proved that as patients and caregivers benefit from a kind of social support it increases their potentials. On the other hand, the lack on this one affects the improvement in patient treatment and the caregiver's faculties. For example, in a study by Peng, et al. [32]. By exploring the social support as linked with other domain of health. The authors found that the patients' social support 
was significantly associated with psychotic symptoms and caregivers burden meaning that the increased subjective social support showed correlation with a lower degree of psychotic symptoms $(\mathrm{p}<.01)$, which was related to a lower level of caregiver burden $(\mathrm{p}<.001)$ [32].

Another interesting study has found the low level of family support in the caregivers of dementia in china. This study reported that the dementia family caregivers experience problems of family functioning associated with the perceived of social support [33].

The integration of social support for caregivers as well as psychiatric patients would be one of to be considered by policy makers and mental health professionals. The social supports is composed by many elements some are easily accessible while other may require means and standard approaches therefore the minimum given in the general society and at the hospital or treatment centers can be adapted.

\subsection{Intervention Care and Mental Health Knowledge for Caregivers of Psychiatric Disorders}

Apart from identified themes that could be seen as focusing on challenges, the literature on caregiving mental health or psychiatric illness in Chinese community shows other important elements that focus on the intervention in caregiving mental illness as well as the addressing mental illness in Chinese community general. One of the intervention on caregiving mental health illness in last ten years have focused in different aspects mainly intervention programs and researches on the proper hypothesized intervention that could work in helping caregivers who are mostly families of psychiatric patients. Therefore, various trials have been explored on this point of view. For instance, the web-based psycho education (iPEP) (www.ipep.hk) was initiated for caregivers of first episode psychosis in Hong Kong. It was made as a solution of providing a comprehensive online resource in both written and video format on knowledge about psychosis and provides the skills of care. In the period of one and half year 809 were registered as members of this platform. The evaluation of its usefulness considered 81 members (10\%) mainly recruited in outpatient services. The majority of the interviewed caregivers reported that the website enhanced their knowledge about psychosis $(85.2 \%)$; increased their understanding of local resources $(77.5 \%)$ and made them feel supported $(74.7 \%)$. Over $80 \%$ of the respondents would recommend the website to others. There was also a general agreement that the website is easy to learn how to operate $(80 \%)$; has clear and easily understandable contents $(81.5 \%)$ and contains sufficient information $(74.1 \%)$. Thus the evaluation of the subset of the members/users of the iPEP suggested that the iPEP program has been well received and appreciated by the caregivers of patients with first episode psychosis locally [34].

Similarly, another randomized controlled trial by Chien, et al. [35] evaluated the effectiveness of the intervention of family psychoeducation and mutual support group on and its implication on the improvements in levels of patient and family functioning and duration of re-hospitalization. The study considered two groups of schizophrenia family caregivers who were randomly assigned to either a family psychoeducation and support group $(n=34)$, or a routine care group $(n=34)$. The Results of multivariate analyses of variance test indicated that the psychoeducation and support group reported greater improvements on family functioning and shorter lengths of patient hospitalizations at the two post-tests (one month and one year after completion of the intervention), as compared to the routine care group [36]. This proves the importance of caregiver's psychoeducation which could be needs to be integrated in the psychiatric management in Chinese community.

The caregiver's literacy about mental health have been seen as an important issue when caring mental health this supports the idea of psychoeducation in caregivers. Although the study by Chen, et al. [37]. Did not evaluate an aspect of outcome of knowledge of the mental health, it is clear that this knowledge would be linked with the outcome of the treatment and well-being in caregivers [37]. The psychoeducation needs to be performed in each technique and approach in order to avoid miss understand which brings worries in caregivers and could influence the burnout. The study of knowledge of Electro conversion therapy ( ETC) shown that caregivers needs necessary information when an approach that concerns them is being used therefore this would be taken in consideration as an intervention technic that would help caregivers and patients in general [38].

The evaluation study on the brief family centered care program for hospitalized patients with bipolar disorders and their family caregivers. Has also shown the benefit of such intervention on both caregivers and the sick relatives. It was seen that this program reduced the caregivers burden and increased the family functioning and has improved health status of caregivers who benefited on this program meanwhile if this program is integrated in mental healthcare management it could help both patients and their caregivers [39].

The intervention was also conducted as home visit partnership two group was involved in this intervention study one was a control group while other was intervention group. Six months after the partnership intervention program, the satisfaction of the experimental group was higher than the control group for several aspects of care. However, the study found that this intervention did not change the care burden. The authors recommended a strengthened mental health home visit as it is helpful for caregivers and patients and finally, they recommend government policies in the reduction of caregivers individual and comprehensive assistance [40].

\section{Discussions}

We conducted this study to have an insight on generalities regarding the challenges of caregiving mental illness in Chinese community. The findings indicate that caregivers are mainly the families of the mentally ill patients and the majority of caregivers are caring for schizophrenic patients. The challenges within caregivers as well as interventions measures were found in this study. Concerning the challenges, the results show indicate that challenges faced by caregivers of mentally ill patients in Chinese community remain an issue of great concern in Chinese 
public health. As stated in detail above these challenges could be grouped in main terms: The High burden of care and burnout, The high stigma of caregivers, The low quality of life in caregivers, The social support. Care and intervention for caregivers of psychiatric disorders.

Although the Chinese community could have its particularities regarding the perception of mental illness and caregiving the mentally ill patients challenges and needs, studies conducted outside China or in other countries show the similarities with those seen in Chinese community. For instance, in a study conducted in Australia by Wancata, et al. [41] show that relieving the social isolation is among the elements with high average among caregivers' needs [41]. In Canada caregivers of patients of Psychiatric illness need social life, tool support, emotional support, information support, and telephone access to the professional treatment team as well as access to experienced counterparts. The lowest percentage of discrimination and fear of labeling could show differences between these two cultures. Which support the point that the social isolation in caregivers of mental illness needs and problems are influenced by the culture. According to the study by Chan chi [42]. Asian countries contrarily to western, due to the issues of honor, people refrain from expressing these problems and tend to hide them and this eventually effect the social support [42].

In terms of caregiver's burden and burnout, a number of studies conducted in other parties of the word show that caregivers of psychiatric patients in china are not unique in presenting the care burden. The caregiver's burden is present in many other countries and was found to be on high and significant level [42, 43]. It was also seen that caregivers of psychiatric can develop other psychological and mental health problems such us anxiety and depression linked with the care they provide to the psychiatric patient [44, 45]. In Africa the study conducted in Zimbabwe found that the psychiatric caregivers experience the high level of burden and burnout [46]. Similarly in a study on African ancestry decedents in south America found the high burden and burnout in caregivers of schizophrenia [47]. In Europe various studies on caregivers' burden were conducted in many countries such as Italy, Spain, Switzerland etc. like in other countries caregivers in these countries presented the burden, for instance in Italy the study nonrelatives of schizophrenic patients identified the burden among them and has called for a supportive intervention for all relatives of schizophrenic patents [48]. Similarly, In a study in Switzerland, [49] identified the burden in caregivers and they found that the most important predictor of burden is the relationship between the caregiver and the patients with exacerbating schizophrenia. This study reported that there are significant changes in the relationship during the acute phase of the illness. Furthermore, the same study showed that threats, nuisances time and restricted social and leisure activities were determinants of high burden in caregivers. As recommended by authors caregivers should lean how to cope with disturbing behaviors of the patient including deciding whether the patient should be admitted to the hospital [49].

The recommendations of the above studies should apply in Chinese community by considering the culture and believes of Chinese society. Some western approaches in managing mental health issues were found to be applicable in Chinese community with some limitation that mostly require some modifications to be adopted in Chinese society $[36,50]$. Another recommendation on the management of the family burden during the caregiving of a chronic mental disorder emerged in study conducted in Sweden. In this study the authors found that the increase of family burden is associated with the increase severity of patient symptoms, and the impaired functioning is a special cause of burden in caregivers. Therefore it was suggested that the professional support to control mental symptoms and improve daily functioning of the patient could help reduce family burden [51]. The same findings were also reported in a study conducted in china and some cases were found lacking a specialized care [23, 52]. Thus, the use of this recommendation could be adopted in any in Chinese community because it sounds helpful and easily applicable in any society includes Chinese community as well as other identified approaches that were recommended in studies conducted in china Chien and Lee [53].

On the point of stigma, psychiatric patients and their caregivers show the stigma most likely in all of its form. In china the stigma is mainly influenced by the culture related factors and the type and severity of the illness and it could be a causing factor for other mental health complications within caregivers and aggravate the severity of disease for the psychiatric sick person [26, 28]. In many other societies the stigma within caregivers was also identified as a matter of concern for mental healthcare therefore like in Chinese society the intervention measures in reducing stigma and its related challenges both in caregivers and psychiatric patients were developed. For instance, in the family education was found to be successful in which the nurse or a professional do a better assessment of family's or caregivers needs in caring for the patient and provide educational particularly to elicit family members' understanding expectations of the illness[54]. This method needs to be strengthened in china due to it simplicity and its outcome .other recommended approaches that could help are for example the emotional support, relief from isolation, receipt of reliable and satisfactory services, information and recognition of their role and contributions [42].

In the field regarding the social support, the studies in Chinese community show critics in overcoming the social support. Most of studies show that the majority of caregivers are adults and they could develop the lack of enough social support in china the most of psychiatric patients live with their families which support the idea that the social support could be given to all family members. The current social support that are given to caregivers is most likely limited to family education or psycho education which still in ongoing programs in different regions of Mainland China. Furthermore, recently in 2016 the Chinese government has initiated a reword policy regarding the care or management of patients with serious mental illness. This policy encourages family caregivers to look after their sick member by preventing them to get involved in criminal activities [14]. Even though this policy was criticized for its risk of causing stigma in psychiatric patients it and their caregivers, if combined with other programs for social support it could be much beneficial and servs as social support tool for both caregivers and patients. Other recommended approach regarding the support is mutual support. This could be performed by creating mutual groups 


\section{Sumerianz Journal of Medical and Healthcare}

which are mainly characterized as client-led interventions to the professional-controlled mental health interventions. This approach involve giving and receiving help and learning to help themselves, as well as sharing experiences and knowledge about common concerns [13]. This approach mainly explored psychiatric patients in China Chien, et al. [35]. The this method sounds useful for caregivers of psychiatric patients if an appropriate program is developed by considering all aspects regarding mental healthcare and caregivers of psychiatric illness. The other type of support that can be recommended is spirituality support for those who have faith. This method was seen useful in some communities such as Iran [55].

In connection with the intervention some studies did not mainly sound as intervention however they have shown points that could help when initiating a collective intervention approach. However other many programs have been developed to intervene in managing caregiving mental illness related issues in Chinese community and was only adopted in identified group and did not benefited by all caregivers in Chinese community as the policies are not developed so far. These interventions are like the educating patients and caregivers on mental health and availing resources that could help in awareness of mental healthcare and caregiving [34]. Creating or initiating the mutual support programs that helps caregivers to overcome dialing burnout burden and other increasing caregivers problems were also found productive in Chinese community [56]. In the same order of idea, the psychoeducation have been found much useful in various communities. the psychoeducation helps caregivers to get skills in problem solving and communication thus it increases caregivers' coping with their situation [57]. It could be recommended to policy makers and mental health workers in Chinese community to think about adding the psychoeducation package in the management of mental illness especially on severe cases like schizophrenia.

\section{Conclusion}

Findings of this study show that caregivers of psychiatric illness in Chinese community face challenges that are in some case different to the rest of the world and much similarities are also found. The high care burden and burnout the stigma in all its form, the issues of social support and issues of low quality of life are the most important challenges found in the literature in the last more than ten years starting 2008. At least there are five main elements need to be considered by policy makers, Health professionals and the general community in dealing with the issues of caregivers of psychiatric illness in Chinese community: first every member of the community especially healthcare professionals should respect and pay attention to the vital role the caregivers of psychiatric illness. This can help in reduce the distress and help in stigma reduction thereby increase their quality of care and the quality of life for both the patient and the caregiver. Second, mental healthcare needs to consider this issue as a serious matter that needs a specific attention. Therefore, mental health professionals are called to conduct further studies in order to inform the policy makers and the general public a very clear evidence regarding the caring mental illness. Third, based on the current evidences the professional healthcare in mental health unities should gain more training on the caring mental illness thereby facilitating them to provide training of proper care, support resources in the society and other skills needed by the caregivers and the community such proper communication as regarding mental illness within the community. Forth the policy makers should understand and consider the problems of caregivers of psychiatric patients and encourage the management programs us presented by professionals therefore, the intervention strategies already proposed should be deeply explored and be used in the settings where needed. Fifth the future studies need to explore whether each psychiatric unity needs a desk for dealing with caregiver's support.

\section{References}

[1] James, S. L., Abate, D., Abate, K. H., Abay, S. M., Abbafati, C., Abbasi, N., and Murray, C. J. L., 2018. "Global, regional, and national incidence, prevalence, and years lived with disability for 354 diseases and injuries for 195 countries and territories, 1990-2017: A systematic analysis for the Global Burden of Disease Study 2017." The Lancet, vol. 392, pp. 1789-1858. Available: https://doi.org/10.1016/S01406736(18)32279-7

[2] Baxter, A. J., Charlson, F. J., Cheng, H. G., Shidhaye, R., Ferrari, A. J., and Whiteford, H. A., 2016. "Prevalence of mental, neurological, and substance use disorders in China and India: a systematic analysis." The Lancet Psychiatry, vol. 3, pp. 832-841. Available: https://doi.org/10.1016/S2215$\underline{0366(16) 30139-0}$

[3] Chan and Yu, I. W., 2004. "Quality of life of clients with schizophrenia." Journal of Advanced Nursing, vol. 45, pp. 72-83. Available: https://doi.org/10.1046/j.1365-2648.2003.02863.x

[4] Thornicroft, G. and Bebbington, P., 1989. "Deinstitutionalisation. From hospital closure to service development." British Journal of Psychiatry, vol. 155, pp. 739-753. Available: https://doi.org/10.1192/bjp.155.6.739

[5] Zendjidjian, X., Richieri, R., Adida, M., Limousin, S., Gaubert, N., Parola, N., and Boyer, L., 2012. "Quality of life among caregivers of individuals with affective disorders." Journal of Affective Disorders, vol. 136, pp. 660-665. Available: https://doi.org/10.1016/j.jad.2011.10.011

[6] Papastavrou, E., Charalambous, A., Tsangari, H., and Karayiannis, G., 2010. "The cost of caring: The relative with schizophrenia." Scandinavian Journal of Caring Sciences, vol. 24, pp. 817-823. Available: https://doi.org/10.1111/j.1471-6712.2010.00782.x

[7] Özen, M., Örüm, M., and Kalenderoğlu, A., 2018. "The burden of schizophrenia on caregivers." Journal of Mood Disorders, p. 1. Available: https://doi.org/10.5455/jmood.20171228112101 


\section{Sumerianz Journal of Medical and Healthcare}

[8] Lauber and Rössler, W., 2007. "Stigma towards people with mental illness in developing countries in Asia." International Review of Psychiatry, vol. 19, pp. 157-178. Available: https://doi.org/10.1080/09540260701278903

[9] Chang, K. H. and Horrocks, S., 2006. "Lived experiences of family caregivers of mentally ill relatives." Journal of Advanced Nursing, vol. 53, pp. 435-443. Available: https://doi.org/10.1111/j.13652648.2006.03732.x

[10] Yip, K. S., 2000. "Have psychiatric services in Hong Kong been impacted by the deinstitutionalization and community care movements?" Administration and Policy in Mental Health, vol. 27, pp. 443-449. Available: https://doi.org/10.1023/A:1021346410494

[11] Song, L. Y., Chang, L. Y., Shih, C. Y., Lin, C. Y., and Yang, M. J., 2005. "Community attitudes towards the mentally ill: The results of a national survey of the Taiwanese population." International Journal of Social Psychiatry, vol. 51, pp. 162-176. Available: https://doi.org/10.1177/0020764005056765

[12] Lo, H. H. M., Ho, W. C., Lau, E. N. S., Lo, C. W., Mak, W. W. S., Ng, S. M., and Leung, C. W. C., 2019. "A brief mindfulness-based family psychoeducation intervention for Chinese young adults with first episode psychosis: A study protocol." Frontiers in Psychology, vol. 10, p. $516 . \quad$ Available: https://doi.org/10.3389/fpsyg.2019.00516

[13] Chien, W. T. and Chan, S. W. C., 2004. "One-year follow-up of a multiple-family-group intervention for Chinese families of patients with schizophrenia." Psychiatric Services, vol. 55, pp. 1276-1284. Available: https://doi.org/10.1176/appi.ps.55.11.1276

[14] Yu, Ph, D., Zhou, W., Ph, D., and Xiao, S., 2016. "China's reward policy for family care of persons with serious mental illness." Psychiatric Services In Advance, Available: https://doi.org/10.1176/appi.ps.201800114

[15] Horey, D. and Canty, J., 2015. "Doing a literature review in nursing, health and social care." Australian Journal of Primary Health, vol. 21, p. 468. Available: https://doi.org/10.1071/pyv21n4_br2

[16] Zhou, Y., Rosenheck, R., Mohamed, S., Ou, Y., Ning, Y., and He, H., 2016. "Comparison of burden among family members of patients diagnosed with schizophrenia and bipolar disorder in a large acute psychiatric hospital in China." BMC Psychiatry, vol. 16, p. 283. Available: https://doi.org/10.1186/s12888-016-0962-y

[17] Zhou, Y., Ning, Y., Rosenheck, R., Sun, B., Zhang, J., Ou, Y., and He, H., 2016. "Effect of living with patients on caregiver burden of individual with schizophrenia in China." Psychiatry Research, vol. 245, pp. 230-237. Available: https://doi.org/10.1016/j.psychres.2016.08.046

[18] Liu, Liu, J., Wang, X. D., Shi, Z., Zhou, Y., Li, J., and Ji, Y., 2018. "Caregiver burden, sleep quality, depression, and anxiety in dementia caregivers: A comparison of frontotemporal lobar degeneration, dementia with Lewy bodies, and Alzheimer's disease." International Psychogeriatrics, vol. 30, pp. 11311138. Available: https://doi.org/10.1017/S1041610217002630

[19] Liu, Wang, L. N., Tan, J. P., Ji, P., Gauthier, S., Zhang, Y. 1., and Liu, S. n., 2012. "Burden, anxiety and depression in caregivers of veterans with dementia in Beijing." Archives of Gerontology and Geriatrics, vol. 55, pp. 560-563. Available: https://doi.org/10.1016/j.archger.2012.05.014

[20] Siu, B. W. M. M., Lam, C. L., and Chan, W. C., 2012. "Pattern and determinants of burden in Chinese families of adults with obsessive-compulsive disorder." Journal of Anxiety Disorders, vol. 26, pp. 252-257. Available: https://doi.org/10.1016/j.janxdis.2011.11.008

[21] Yu, Chen, J., Hu, J., and Hu, J. C., 2019. "Relationship between mental health and burden among primary caregivers of outpatients with schizophrenia." Family Process, vol. 58, pp. 370-383. Available: https://doi.org/10.1111/famp.12340

[22] He, H., Ning, Y., Rosenheck, R., Sun, B., Zhang, J., and Zhou, Y., 2015. "Is severity of family burden a correlate of length of stay?" Psychiatry Research, vol. 230, pp. 84-89. Available: https://doi.org/10.1016/j.psychres.2015.08.024

[23] Yu, Liu, Z. w., Tang, B. W., Zhao, M., Liu, X. G., and Xiao, S. Y., 2017. "Reported family burden of schizophrenia patients in rural China." PLoS ONE, vol. 12, Available: https://doi.org/10.1371/journal.pone.0179425

[24] Yu, Tang, B. W., Liu, Z. W., Chen, Y. M., Zhang, X. Y., and Xiao, S., 2018. "Who cares for the schizophrenia individuals in rural China - A profile of primary family caregivers." Comprehensive Psychiatry, vol. 84, pp. 47-53. Available: https://doi.org/10.1016/j.comppsych.2018.04.002

[25] Chen, Zhao, Y., Tang, J., Jin, G., Liu, Y., Zhao, X., and Lu, X., 2019. "The burden, support and needs of primary family caregivers of people experiencing schizophrenia in Beijing communities: A qualitative study." BMC Psychiatry, vol. 19, Available: https://doi.org/10.1186/s12888-019-2052-4

[26] Wong, Kong, D., Tu, L., and Frasso, R., 2018. "My bitterness is deeper than the ocean": Understanding internalized stigma from the perspectives of persons with schizophrenia and their family caregivers." International Journal of Mental Health Systems, vol. 12, p. 14. Available: https://doi.org/10.1186/s13033018-0192-4

[27] Huang, X. Y., Sun, F. K., Yen, W. J., and Fu, C. M., 2008. "The coping experiences of carers who live with someone who has schizophrenia." Journal of Clinical Nursing, vol. 17, pp. 817-826. Available: https://doi.org/10.1111/j.1365-2702.2007.02130.X

[28] Hsiao, C. Y., Lee, C. T., Lu, H. L., and Tsai, Y. F., 2017. "Living with schizophrenia: Health-related quality of life among primary family caregivers." Journal of Clinical Nursing, vol. 26, pp. 5151-5159. Available: https://doi.org/10.1111/jocn.14063 


\section{Sumerianz Journal of Medical and Healthcare}

[29] Chen, Chang, W. C., Hui, C. L. M., Chan, S. K. W., Lee, E. H. M., and Chen, E. Y. H., 2016. "Self-stigma and affiliate stigma in first-episode psychosis patients and their caregivers." Social Psychiatry and Psychiatric Epidemiology, vol. 51, pp. 1225-1231. Available: https://doi.org/10.1007/s00127-016-1221-8

[30] Guan, L., Xiang, Y., Ma, X., Weng, Y., and Liang, W., 2016. "Qualities of life of patients with psychotic disorders and their family caregivers: Comparison between hospitalised and community-based treatment in beijing, China." PloS One, vol. 11, p. e0166811. Available: https://doi.org/10.1371/journal.pone.0166811

[31] Wong, Lam, A. Y. K., Chan, S. K., and Chan, S. F., 2012. "Quality of life of caregivers with relatives suffering from mental illness in Hong Kong: roles of caregiver characteristics, caregiving burdens, and satisfaction with psychiatric services." Health and Quality of Life Outcomes, vol. 10, p. 15. Available: https://doi.org/10.1186/1477-7525-10-15

[32] Peng, M. M., Zhang, T. M., Liu, K. Z., Gong, K., Huang, C. H., Dai, G. Z., and Ran, M. S., 2019. "Perception of social support and psychotic symptoms among persons with schizophrenia: A strategy to lessen caregiver burden." International Journal of Social Psychiatry, vol. 65, pp. 548-557. Available: https://doi.org/10.1177/0020764019866230

[33] Zhang, Xiong, R. H., Sara, H., Zhang, J. J., and Zhang, X. Q., 2013. "Psychological distress, family functioning, and social support in family caregivers for patients with dementia in the mainland of China." Chinese Medical Journal, vol. 126, pp. 3417-3421. Available: https://doi.org/10.3760/cma.j.issn.0366$\underline{6999.20131254}$

[34] Chan, Tse, S., Sit, H. L. T., Hui, C. L. M., Lee, E. H. M., Chang, W. C., and Chen, E. Y. H., 2016. "Webbased psychoeducation program for caregivers of first-episode of psychosis: An experience of chinese population in Hong Kong." Frontiers in Psychology, Available: https://doi.org/10.3389/fpsyg.2016.02006

[35] Chien, W. T., Thompson, D. R., and Norman, I., 2008. "Effects of a mutual support group for families of Chinese people with schizophrenia: ophre ia 18-month follow-up." American Journal of Community Psychology, vol. 42, pp. 122-134. Available: https://doi.org/10.1007/s10464-008-9178-8

[36] Chien, W. T., 2008. "Effectiveness of psychoeducation and mutual support group program for family caregivers of chinese people with schizophrenia." The Open Nursing Journal, vol. 2, pp. 28-39. Available: https://doi.org/10.2174/1874434600802010028

[37] Chen, Wu, Q., Qi, C., Deng, H., Wang, X., He, H., and Liu, T., 2017. "Mental health literacy about schizophrenia and depression: A survey among Chinese caregivers of patients with mental disorder." BMC Psychiatry, vol. 17, p. 89. Available: https://doi.org/10.1186/s12888-017-1245-y

[38] Zhang, Zhou, F. C., Zhang, L., Ng, C. H., Ungvari, G. S., Wang, G., and Xiang, Y. T., 2018. "Knowledge and attitudes of older psychiatric patients and their caregivers towards electroconvulsive therapy." Psychogeriatrics: The Official Journal of the Japanese Psychogeriatric Society, vol. 18, pp. 343-350. Available: https://doi.org/10.1111/psyg.12326

[39] Lee, H. J., Lin, E. C. L., Chen, M. B., Su, T. P., and Chiang, L. C., 2018. "Randomized, controlled trial of a brief family-centred care programme for hospitalized patients with bipolar disorder and their family caregivers." International Journal of Mental Health Nursing, vol. 27, pp. 61-71. Available: https://doi.org/10.1111/inm.12294

[40] Cheng, Huang, X. Y., Lin, M. J., Wang, Y. H., and Yeh, T. P., 2018. "The influence of a mental health home visit service partnership intervention on the caregivers' home visit service satisfaction and care burden." Journal of Clinical Nursing, vol. 27, pp. e668-e677. Available: https://doi.org/10.1111/jocn.14123

[41] Wancata, J., Krautgartner, M., Berner, J., Scumaci, S., Freidl, M., Alexandrowicz, R., and Rittmannsberger, H., 2006. "The "Carers" needs assessment for Schizophrenia". An instrument to assess the needs of relatives caring for schizophrenia patients." Social Psychiatry and Psychiatric Epidemiology, vol. 41, pp. 221-229. Available: https://doi.org/10.1007/s00127-005-0021-3

[42] Chan chi, 2011. "Global perspective of burden of family caregivers for persons with schizophrenia." Archives of Psychiatric Nursing, vol. 25, pp. 339-349. Available: https://doi.org/10.1016/j.apnu.2011.03.008

[43] Ogilvie, A. D., Morant, N., and Goodwin, G. M., 2005. "The burden on informal caregivers of people with bipolar disorder." Bipolar Disorders, Supplement, vol. 7, pp. 25-32. Available: https://doi.org/10.1111/j.1399-5618.2005.00191.x

[44] Steele, A., Maruyama, N., and Galynker, I., 2010. "Psychiatric symptoms in caregivers of patients with bipolar disorder: A review." Journal of Affective Disorders, vol. 121, pp. 10-21. Available: https://doi.org/10.1016/j.jad.2009.04.020

[45] Cuijpers, P. and Stam, H., 2000. "Burnout among relatives of psychiatric patients attending psychoeducational support groups." Psychiatric Services, vol. 51, pp. 375-379. Available: https://doi.org/10.1176/appi.ps.51.3.375

[46] Marimbe, B. D., Cowan, F., Kajawu, L., Muchirahondo, F., and Lund, C., 2016. "Perceived burden of care and reported coping strategies and needs for family caregivers of people with mental disorders in Zimbabwe." African Journal of Disability, vol. 5, Available: https://doi.org/10.4102/ajod.v5i1.209

[47] Alexander, G., Bebee, C. E., Chen, K. M., Vignes, R. M., Des, D., B., Escoffery, R., and Jackson, M. D., 2016. "Burden of caregivers of adult patients with schizophrenia in a predominantly African ancestry population." Quality of Life Research, vol. 25, pp. 393-400. Available: https://doi.org/10.1007/s11136-015$\underline{1077-5}$ 


\section{Sumerianz Journal of Medical and Healthcare}

[48] Magliano, L., Fiorillo, A., Malangone, C., Marasco, C., and Guarneri, M. M. M. N. M. H. P. W. G., 2003. "The effect of social network on burden and pessimism in relatives of patients with schizophrenia." Orthopsychiatry, vol. 73, pp. 302-309. Available: https://doi.org/10.1037/0002-9432.73.3.302

[49] Lauber, Eichenberger, A., Luginbühl, P., Keller, C., and Rössler, W., 2003. "Determinants of burden in caregivers of patients with exacerbating schizophrenia." European Psychiatry, vol. 18, pp. 285-289. Available: https://doi.org/10.1016/j.eurpsy.2003.06.004

[50] Li, W., Zhang, L., Luo, X., Liu, B., Liu, Z. Z., Lin, F., and Naeem, F., 2017. "A qualitative study to explore views of patients', carers' and mental health professionals' to inform cultural adaptation of CBT for psychosis (CBTp) in China." BMC Psychiatry, vol. 17, p. 131. Available: https://doi.org/10.1186/s12888$\underline{017-1290-6}$

[51] Hjärthag, F., Helldin, L., Karilampi, U., and Norlander, T., 2010. "Illness-related components for the family burden of relatives to patients with psychotic illness." Social Psychiatry and Psychiatric Epidemiology, vol. 45, pp. 275-283. Available: https://doi.org/10.1007/s00127-009-0065-X

[52] Wu, C., Gao, L., Chen, S., and Dong, H., 2016. "Care services for elderly people with dementia in rural China: a case study." Bulletin of the World Health Organization, vol. 94, pp. 167-173. Available: https://doi.org/10.2471/BLT.15.160929

[53] Chien, W. T. and Lee, I. Y. M., 2010. The schizophrenia care management program for family caregivers of Chinese patients with schizophrenia vol. 61. Washington, D.C.: Psychiatric Services. pp. 317-320.

[54] Cheng and Chan, S., 2005. "Psychoeducation program for chinese family carers of members with schizophrenia 1." Western Journal of Nursing Research, vol. 27, Available: https://doi.org/10.1177/0193945905275938

[55] Mohammad, A., Mousa, A., and Alireza, I. J. M., 2018. "Challenges of family caregivers of patients with mental disorders in Iran: A narrative review." Iranian Journal of Nursing and Midwifery Research, vol. 23, Available: https://doi.org/10.4103/ijnmr.IJNMR 122 17

[56] Ran, M. S., Xiang, M. Z., Chan, C. L. W., Leff, J., Simpson, P., Huang, M. S., and Li, S. G., 2003. "Effectiveness of psychoeducational intervention for rural Chinese families experiencing schizophrenia--a randomised controlled trial." Social Psychiatry and Psychiatric Epidemiology, vol. 38, pp. 69-75. Available: https://doi.org/10.1007/s00127-003-0601-Z

[57] Xia, J., Merinder, L. B., and Belgamwar, M. R., 2011. "Psychoeducation for schizophrenia." Schizophrenia Bulletin, vol. 37, pp. 21-22. Available: https://doi.org/10.1093/schbul/sbq138 